\title{
Progressive Respiratory Distress in a Patient with Splenic Marginal Zone Lymphoma
}

\author{
Azuchi Masuda, Yasushi Isobe, Koichi Sugimoto, Jun Ando and Kazuo Oshimi
}

Key words: splenic marginal zone lymphoma, endobronchial tumors, respiratory distress, computed tomography

(Inter Med 47: 1281-1282, 2008)

(DOI: 10.2169/internalmedicine.47.0957)
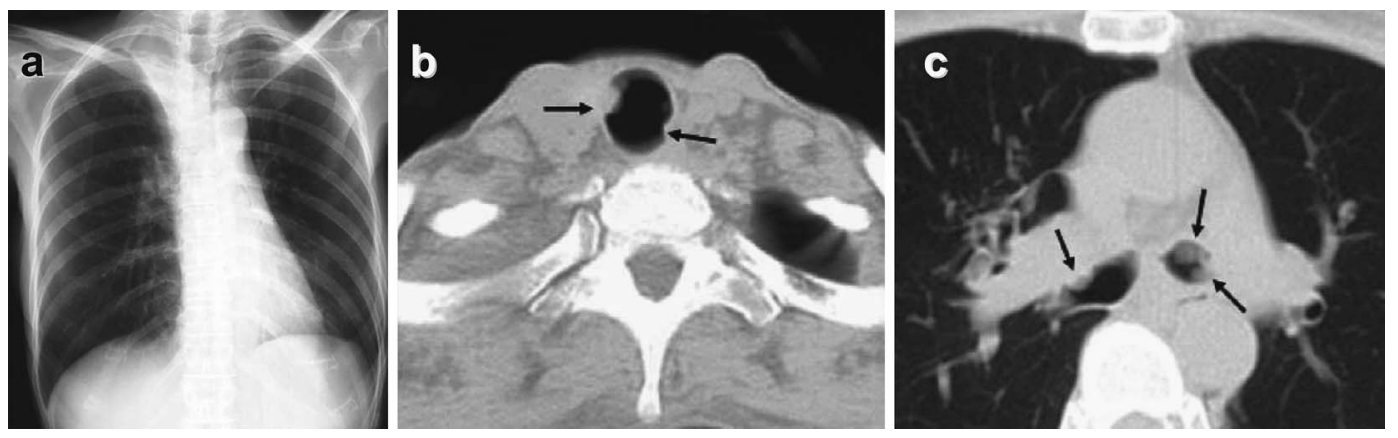

Picture 1. Radiological findings prior to chemotherapy. Chest X-ray showed atelectasis at the right upper lung field (a). Computed tomography detected multiple endobronchial tumors (arrows) (b), (c).
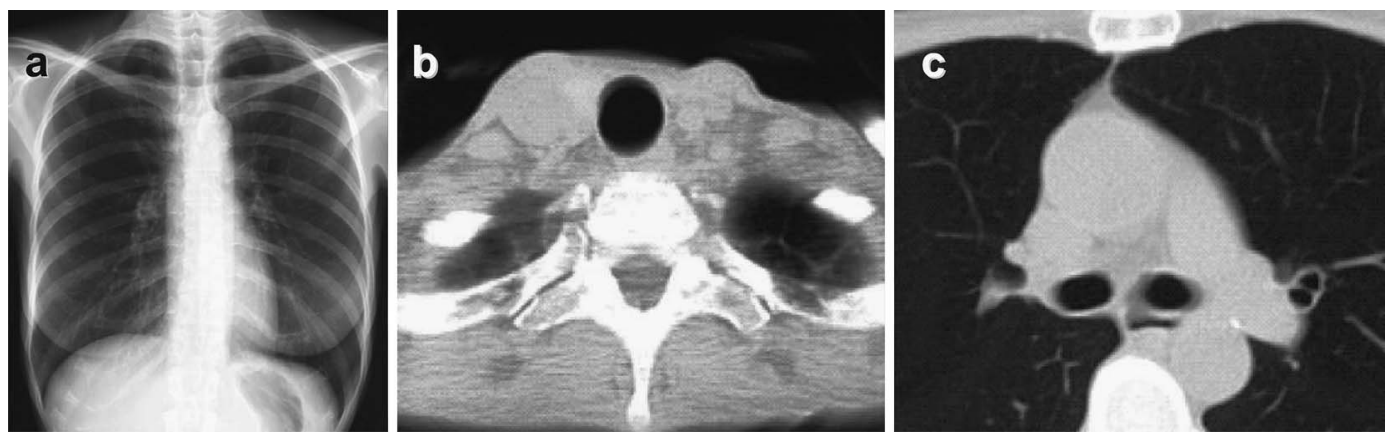

Picture 2. Radiological findings after 1 course of CHOP chemotherapy. Chest X-ray did not reveal any particular findings (a). Endobronchial tumors disappeared after the chemotherapy (b), (c).

\section{Short Summary}

A 53-year-old Japanese woman was referred to our hospital because of fever, fatigue, pancytopenia and splenomegaly. The patient was diagnosed with splenic mar- ginal zone lymphoma after undergoing splenectomy in February 2000. Thereafter, she was free from any symptoms until she developed progressive dyspnea in November 2005. Chest X-ray showed the presence of pulmonary atelectasis at the right upper lung field (Picture 1a). Computed tomography (CT) demonstrated airway obstruction caused by multi- 
ple endobronchial tumors in her primary bronchus and bilateral main bronchi (Pictures 1b, 1c). We detected elevated serum levels of soluble forms of interleukin-2 receptor. Recurrence of lymphoma was strongly suspected. Although a bronchoscopic study was planned, her respiratory distress became so severe that she needed immediate combination chemotherapy consisting of cyclophosphamide, doxorubicin, vincristine, and prednisolone (CHOP). Her symptoms were dramatically resolved after the chemotherapy. X-ray and CT confirmed disappearances of atelectasis and endobronchial tumors (Pictures 2a, 2b, 2c). Although endobronchial tumor presentation is extremely rare in non-Hodgkin's lymphomas $(1,2)$, we believe that these tumors represent the recurrence of the lymphoma. She subsequently received five courses of rituximab plus CHOP chemotherapy and remained well without any symptoms or findings related to lymphoma in March 2008. In this case, CT was helpful to evaluate endobronchial tumors in the emergent situation.

This work was not supported by any grants.

\section{References}

1. Eng J, Sabanathan S. Endbronchial non-Hodgkin's lymphoma. J Cardiovasc Surg 34: 351-354, 1993.

2. Solomonov A, Zuckerman T, Goralnik L, et al. Non-Hodgkin's lymphoma presenting as an endobronchial tumor: Report of eight cases and literature review. Am J Hematol 2008 [Epub ahead of print].

(C) 2008 The Japanese Society of Internal Medicine http://www.naika.or.jp/imindex.html 